\title{
A smart microfluidic platform for rapid multiplexed detection of foodborne pathogens
}

\section{Azinheiro, Sarah}

2020-08

Azinheiro , S , Kant , K , Shahbazi , M-A , Garrido-Maestu , A , Prado , M \& Dieguez , L 2020

, ' A smart microfluidic platform for rapid multiplexed detection of foodborne pathogens ' ,

Food Control , vol. 114 , 107242 . https://doi.org/10.1016/j.foodcont.2020.107242

http://hdl.handle.net/10138/328169

https://doi.org/10.1016/j.foodcont.2020.107242

cc_by_nc_nd

acceptedVersion

Downloaded from Helda, University of Helsinki institutional repository.

This is an electronic reprint of the original article.

This reprint may differ from the original in pagination and typographic detail.

Please cite the original version. 


\section{Journal Pre-proof}

A smart microfluidic platform for rapid multiplexed detection of foodborne pathogens

Sarah Azinheiro, Krishna Kant, Mohammad-Ali Shahbazi, Alejandro Garrido-Maestu, Marta Prado, Lorena Dieguez

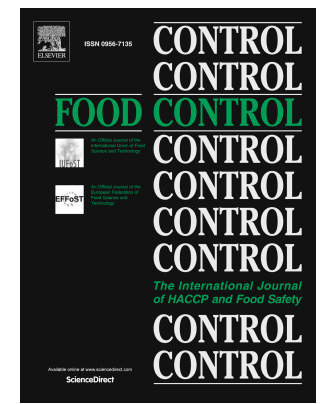

PII: S0956-7135(20)30158-4

DOI: https://doi.org/10.1016/j.foodcont.2020.107242

Reference: JFCO 107242

To appear in: Food Control

Received Date: 10 January 2020

Revised Date: 12 March 2020

Accepted Date: 13 March 2020

Please cite this article as: Azinheiro S., Kant K., Shahbazi M.-A., Garrido-Maestu A., Prado M. \& Dieguez L., A smart microfluidic platform for rapid multiplexed detection of foodborne pathogens, Food Control (2020), doi: https://doi.org/10.1016/j.foodcont.2020.107242.

This is a PDF file of an article that has undergone enhancements after acceptance, such as the addition of a cover page and metadata, and formatting for readability, but it is not yet the definitive version of record. This version will undergo additional copyediting, typesetting and review before it is published in its final form, but we are providing this version to give early visibility of the article. Please note that, during the production process, errors may be discovered which could affect the content, and all legal disclaimers that apply to the journal pertain.

() 2020 Published by Elsevier Ltd. 


\section{Credit Author Statement:}

The authors of this publication have contributed to this work as follows:

Conceptualization-K. K., M-A S., Methodology-S. A., K. K., Validation-Sarah Azinheiro, Krishna Kant, A. G-M., Formal analysis-S. A., K. K., Investigation- K. K., S. A., Resources- A. G-M., M. P., L. D. Data curation- S. A., K. K., Writing original draft preparation- S. A., K. K., Review and editing- S. A., K. K., A. G-M., Project administration- M. P., L. D., Funding acquisition- M. P., L. D. 


\title{
A smart microfluidic platform for rapid multiplexed detection of
}

\section{foodborne pathogens}

\author{
Sarah Azinheiro $^{{ }^{*}}$, Krishna Kant ${ }^{1 * \#}$, Mohammad-Ali Shahbazi ${ }^{2}$, Alejandro Garrido-Maestu ${ }^{l}$, Marta \\ Prado $^{l}$, Lorena Dieguez ${ }^{1}$
}

(1)

${ }^{1}$ Department of Life Sciences, International Iberian Nanotechnology Laboratory (INL), Braga, Portugal

${ }^{2}$ Division of Pharmaceutical Chemistry and Technology, Faculty of Pharmacy, University of Helsinki, Finland

*Authors contributed equally

\#corresponding author: krishna.kant@inl.int

sarah.azinheiro@inl.int

krishna.kant@inl.int

m.a.shahbazi@helsinki.fi

alejandro.garrido@inl.int

marta.prado@inl.int

lorena.dieguez@inl.int 
Abstract:

Rapid and sensitive detection of foodborne pathogens in food industry is of high importance in dayto-day practice to ensure safe food. To address this issue, multiple foodborne pathogens are targeted for rapid identification based in DNA amplification. A 3D PDMS sponge was fabricated using salt crystals as scarifying mold and functionalized with a ligand, apolipoprotein- $\mathrm{H}(\mathrm{ApoH})$, to test bacterial capturing for both Gram positive (L. monocytogenes) and negative bacteria (Salmonella spp.), in a microfluidic device. Pure culture of both pathogens in a range of $\sim 10$ to $10^{5} \mathrm{CFU} / \mathrm{mL}$ were tested and the application of the developed automated pre-concentration protocol in real samples was verified using spiked surface samples after swab sampling. Bacterial DNA was extracted directly from the sponge and used for Real Time quantitative Polymerase Chain Reaction (qPCR) detection. The sponges did not show any significant resistance to sample flow and could easily be incorporated in a microfluidic device. A capture efficiency above $70 \%$ was observed for both targeted (Gram positive and Gram negative) pathogens and a Limit of Detection (LoD) in the range of $10^{3}$ and $10^{4}$ CFU/mL was obtained for Salmonella spp. and L. monocytogenes, respectively. Using this approached, we are able to perform multiplexed (Gram positive and Gram negative) capturing and reduce the enrichment time compared to the gold standard plate culture (over 1-day) method. The use of a 3D sponge for direct capturing of multiplexed pathogen on microfluidic device, followed by qPCR detection is an efficient and versatile method to stratify the presence of bacteria. This approach and methodology has potential to be integrated in full automatized device and used as point of need (PoN) system for foodborne pathogen stratification in food packaging/production industries.

Keywords: 3D sponge, foodborne pathogen, microfluidic device, multiplexed detection, qPCR. 
Contamination of food and water with bacteria is currently a significant public concern worldwide, as

it is associated with increased mortality rate and economic burden due to the possible outbreaks.

Common symptomatology make difficult to identify the causative pathogen. Therefore, there is a need for tighter monitoring of food and water to decrease global incidence of foodborne diseases and their related crucial safety issues. To highlight its importance, it is worth noting that although the US Center for Disease Control and Prevention (CDC) 2018 has estimated nearly 3000 annual deaths in US due to the contamination of food and drinking water, which can be considered underestimated as a result of misdiagnosis and improper sample collection and testing (Dewey-Mattia et al. 2018). Among the causative agents of food poisoning, L. monocytogenes is one of the most problematic foodborne pathogen, with one of the highest mortality rate of 13.8\%, reported in 2017 in European, and Salmonella spp. continue to be the second commonest reported zoonotic agent, responsible for many hospitalizations (EFSA and ECDC 2018; Vidic et al. 2019). Traditional methods for the detection of these bacteria in food matrix are based on sample enrichment and subsequent pathogen culturing on agar plate, followed by biochemical identification (Cai, Singh, and Sharma 2007; Wang and Duncan 2017). This method has limitations related to the requirement of specific media for enrichment, optimized incubation temperatures for different bacterial species, high number of Petri dishes for culturing, and a trained personnel for isolation and identification of the microorganisms (Vinayaka et al. 2019). Moreover, successful identification of a specific pathogen may take up to one week, which is considered lengthy. Therefore, such conventional gold standards microbiological methods do not meet the demand of rapid pathogen testing in food products. A new branch of on-site analytical detection with the potential to overcome weaknesses of classical methods combines microfluidics, chemical engineering, and biosensors (Liu et al. 2019)(Xu. 2019). Such portable sensors have attracted an exponential plethora of attention in the last decade for food and water quality control by getting the benefits of polymerase chain reaction (PCR) to detect specific microbial DNA as a target 
not culturable even in their viable form, leading to a false negative result using culturing methods (Dao et al. 2018). Therefore, combining PCR with a lab-on-a-chip device prevents this risk while rendering rapid detection to the system, if a proper pre-concentration strategy is applied in the device. The attachment of the bacteria within the microfluidic device happen through its tendency to capturing ligands available on the surface of the chamber where the food sample is passed. Although it is considered laborious and expensive, adding a pre-concentration step is a necessary step to achieve an optimal DNA quantity when low numbers of bacteria are presents in food samples. Besides, it is crucial to use a universal capturing ligand in pre-concentration step since multiple pathogens may be present in the same food sample.

Herein, we have developed a miniaturized micro-device for rapid multiplexed (gram positive and gram negative) detection of foodborne pathogens with an automated pre-concentration protocol using universal capturing ligand apolipoprotein- $\mathrm{H}(\mathrm{ApoH})$ functionalized 3D sponges in microfluidic device. The 3D sponge was developed using PDMS with defined pore size and porosity for uninterrupted flow of the food sample to perform the function of bacteria pre-concentration step. The DNA extraction and purification from captured bacteria was performed directly from the sponge and followed by qPCR quantification.

\section{Experimental Section:}

\subsection{Reagents and material}

All chemicals were purchased at Sigma-Aldrich unless otherwise specifically stated. Milli-Q ultrapure water (Millipore, USA) was used throughout all the experiments.

\subsection{Methods}

\subsubsection{Fabrication of PDMS sponge}

A controlled size $(80-100 \mu \mathrm{m})$ of salt $(\mathrm{NaCl}$, Sigma-Aldrich), particles (i.e., granulated, sanding particles) were prepared to develop salt template for 3D PDMS sponge. The powdered salt particles were rubbed by adding water and then cast onto molds (empty syringe) to shape the template for PDMS sponge. Thereafter, the molds were carefully cut away to recover the solidified PDMS cylinder. The salt used in mold were placed into an empty syringe in which a mixture of PDMS 
112 (Sylgard 184) with curing agent in ration of (10: 1) by weight was poured. The syringe was degassed

113 by connected with a vacuum line so the PDMS can infiltrated into the salt templates through capillary

114 or air gaps under vacuum forces. The salt templates with the absorbed mixture were then cured at 65

$115{ }^{\circ} \mathrm{C}$ for 4 hours. After the curing process, the salt templates were dissolved and washed away by 116 soaking them in a water bath under continuous steering. Finally, after the removal of the salt templates, 3D interconnected microporous PDMS sponges were formed (Fig. 1).

\subsubsection{Surface modification of Sponge}

119

120

To functionalize the surface of the 3D PDMS structures for bacterial capturing, a multi-step protocol was performed to bind ligands on the fabricated sponges. Initially, surface oxygen plasma treatment was performed on PDMS sponge. To do so, the PDMS surface was first cleaned with isopropanol, dried, and then treated with oxygen plasma for 2 min under vacuum using plasma cleaner (Harrick, Germany). Right after surface hydroxylation, the samples were immersed in a freshly prepared $5 \% \mathrm{v} /$ v solution of (3-Aminopropyl) triethoxysilane in ethanol, and incubated for $150 \mathrm{~min}$ at RT. The samples were then washed gently with ethanol, cured at $80^{\circ} \mathrm{C}$ for $1 \mathrm{~h}$, and sonicated in ethanol for 10 $\mathrm{s}$ to remove the physically adsorbed and unbound (3-Aminopropyl) triethoxysilane molecules. Rinsing with ethanol was again performed in the next step and then the samples were blown dry with a nitrogen stream. The obtained epoxide functionalized PDMS structures were then stored in a sealed container at room temperature until use. After surface modification the sponges were functionalized with $5 \mu \mathrm{g} / \mathrm{mL}$ of ApoH protein (ApoH-Technologies, France) or $10 \mu \mathrm{g} / \mathrm{mL}$ of anti- $L$. monocytogenes antibody (Ab) (MAB8953, Abnova, Taiwan) depending on the experiment. Two hundred microliters of the solution were add to a tube with the sponge, vortexed vigorously and incubated overnight $(\mathrm{ON})$ at $4{ }^{\circ} \mathrm{C}$ to allow the binding of the protein or the antibody to the sponge. The sponge was then washed three times with PBS $\left(137 \mathrm{mM} \mathrm{NaCl}, 12 \mathrm{mM}\right.$ Phosphate $\left(\mathrm{Na}_{2} \mathrm{HPO}_{4} /\right.$ $\left.\left.\mathrm{KH}_{2} \mathrm{PO}_{4}\right), 2.7 \mathrm{mM} \mathrm{KCl}, \mathrm{pH} 7.4\right)$, and stored at $4{ }^{\circ} \mathrm{C}$ until use.

\subsubsection{Sponge characterization}


Different approaches were used to ensure the modification of the PDMS sponge and the capturing of

138 the bacteria into the pores. Fourier Transform Infrared Spectroscopy (FTIR) (VERTEX 80v vacuum,

139 Bruker) characterization was carried out on surface modifies PDMS sponge to check the availability

140 of the functional groups over the surface of the pores of sponge. All the absorption spectra were acquired from 500 to $4000 \mathrm{~cm}^{-1}$ with 48 scans and resolution of $0.2 \mathrm{~cm}^{-1}$, a baseline-correction was also performed prior to the analysis. To confirm the epoxide modification of the PDMS, and the binding of the ligand, a fluorescence detection of the ligand was performed. The sponge was functionalized for $24 \mathrm{~h}$ with the anti- L. monocytogenes antibody complexed with a secondary antibody conjugate Atto-633 dissolved at a concentration of $1 \mathrm{mg} / \mathrm{mL}$ in PBS (pH 7.4). The fluorescence of the bounded antibody over the PDMS was monitored using inverted fluorescence microscopy (Nikon Ti-E).

Scanning Electron Microscopy (SEM) studies were performed on $S$. Typhimurium and $L$. monocytogenes bonded on 3D PDMS sponge functionalized with ApoH protein. Prior to the SEM studies, the sample was fixed according to the standard protocol for cultured microorganisms with slight modifications (Sousa et al. 2015). Briefly, a pure culture of target bacteria was added to the sponge and fixed with $2.5 \%$ glutaraldehyde solution for $1 \mathrm{~h}$. After fixation, the sponges were washed three times with mili-Q water and dehydrated later, incubating the sponge at $37^{\circ} \mathrm{C}$, until completely dry. The dried sample was used for SEM imaging. The SEM imaging was done with 5 - $10 \mathrm{KV}$ beam line (SEM, Quanta 650 FEI) under high vacuum conditions.

\subsubsection{Preparation of bacterial samples and capturing on sponge}

For all experiments performed, L. monocytogenes (WDCM 00021) and S. Typhimurium (WDCM 00031) were used as reference strain. A fresh culture was prepared adding an isolated colony to $4 \mathrm{~mL}$ of Nutrient Broth (NB, Biokar Diagnostics S.A., France) and incubated ON at $37^{\circ} \mathrm{C}$. Ten-fold serial dilution were performed in PBS to achieve the desired bacterial concentration. The bacterial concentration spiked in each experiment was obtained by plating two dilution on Tryptic Soy Yeast 
specially designed microfluidic device was fabricated to incorporate the PDMS sponge with the fluidic connections as presented in Fig. 2. The bacterial solution was flow through the sponge with controlled flow rate of $10 \mu \mathrm{L} / \mathrm{min}$. A washing step with $800 \mu \mathrm{L}$ of PBS at the same flow rate was performed to recover all bacteria cells unbounded to the sponge. The outlet solution was collected and used for culture plate counting to confirm the efficiency of bacterial capturing in sponge. After the sample solution passed through the sponge, DNA extraction from sponge was performed for qPCR analysis.

\section{Detection by qPCR}

To evaluate the ability of the device to concentrate L. monocytogenes and Salmonella spp. cells, being possible their detection by DNA amplification techniques, the DNA extraction directly from the sponge was performed followed by qPCR reaction.

\subsubsection{DNA extraction}

After passing the bacterial cultures through the device, and washing step, the PDMS sponge was removed and transfer to a $2 \mathrm{~mL}$ tube to proceed with the DNA extraction, which was performed based on Lysis-GuSCN method described by Kawasaki et al (Kawasaki et al. 2005) with several modifications. For this step the lysis was performed directly in the sponge adding $200 \mu \mathrm{L}$ of an enzymatic solution containing $1 \mathrm{mg} / \mathrm{mL}$ of achromopeptidase and $20 \mathrm{mg} / \mathrm{mL}$ of lysozyme in TE $2 \mathrm{X}$ (20 mM Tris-HCl, 2 mM EDTA, pH 8) with $1.2 \%$ of Triton X-100 and vortexed vigorously. The lysis was performed at $37 \square \mathrm{C}$, during $30 \mathrm{~min}$ with constant agitation (1400 rpm). After incubation, $300 \mu \mathrm{L}$ of a buffer with $4 \mathrm{M}$ of Guanidine thiocyanate, and $1 \%$ of Tween 20 , were added, and $400 \mu \mathrm{L}$ of this solution were transfer to a new tube containing $400 \mu \mathrm{L}$ of $100 \%$ isopropanol. The mixture was vortexed and centrifuged at $16000 \mathrm{x}$ g for $10 \mathrm{~min}$. The pellet was washed with $75 \%$ isopropanol and resuspended in $160 \mu \mathrm{L}$ of Mili-Q water and incubated at $70^{\circ} \mathrm{C}, 3 \mathrm{~min}$. Finally, the DNA extract was centrifuged $5 \mathrm{~min}$ at $16000 \mathrm{x}$ g, to separate remaining cellular debris.

\subsubsection{2. qPCR}


The qPCR reaction was performed using hydrolysis probes. The probes were designed to hybridize with the specific fragment amplified by the respective primers. The primers and probes used in this study were designed to specifically detect L. monocytogenes and Salmonella spp. and the use of a non-competitive internal amplification control (NC-IAC) was added to ensure the reliability of the results, ruling out false negative results due to reaction inhibition, identified as no amplification of the NC-IAC

The targeted genes chosen for the identification of L. monocytogenes and Salmonella spp. were actA and $t t r$ gene respectively. $t$ tr primers and probe used in these experiments were design and evaluated in a previous study (Garrido-Maestu et al. 2017), but those targeting actA were newly designed with Primer3Plus (Untergasser et al. 2012), setting as template the consensus sequence generated after the alignment of the target sequences with CLC Sequence Viewer (C L C Bio-Qiagen 2016). The gene actA has been extensively used to detect $L$. monocytogenes by qPCR (Cai et al. 2002; Coroneo et al. 2016; Oravcová, Kuchta, and Kaclíková 2007). The newly designed primers and probe were evaluated in terms of inclusivity and exclusivity against the panel of microorganisms provided in Supplementary material Table S1, and after confirmation of performance, the amplification efficiency was also determined and presented in Supplementary material Fig. S1.

NC-IAC was previously designed and implemented as described in Garrido $\square$ Maestu et al. 2019 (Garrido $\square$ Maestu et al. 2019). The DNA sequence for the NC-IAC was designed generating a random sequence and then used as template for the primers and probe design. All primers and probe sequence and concentration used are shown in Supplementary material Table S2. The reaction was performed in a final volume of $20 \mu \mathrm{L}$ and $3 \mu \mathrm{L}$ of sample with $10 \mu \mathrm{L}$ of TaqMan ${ }^{\mathrm{TM}}$ Fast Advanced Master Mix (ThermoFisher, USA) and $1 \mu \mathrm{L}$ of NC-IAC DNA ( 926 copies/ $\mu \mathrm{L})$. The thermal profile used was the recommended by the manufacturer for the fast format, with an optimized annealing/ extension temperature. A step for UDG treatment at $50 \square \mathrm{C}$ for 2 min was first performed, followed by a hotstart activation of the polymerase at $95 \square \mathrm{C}$ during for $2 \mathrm{~min}$, and 40 cycles of $95 \square \mathrm{C}$ for $1 \mathrm{~s}$ and 63 $\square \mathrm{C}$ for $20 \mathrm{~s}$.

\subsubsection{Methodology evaluation}


To evaluate the capacity of the device with the PDMS sponge to concentrate the bacteria, the capture efficiency was obtained by plating the flow-through recovered from the device outlet. To determine the concentration of bacteria released, serial dilutions were made in PBS and plated on COMPASS and XLD for the isolation of L. monocytogenes and Salmonella spp. respectively. The capture efficiency was calculated using the following equation:

\section{Capture efficiency $(\%)=(\mathrm{Nt}-\mathrm{Ne}) / \mathrm{Nt} \times 100$}

Where Nt is the number of bacterial cells in the sample, introduce in the device and $\mathrm{Ne}$ is the number of uncaptured bacterial cells, recovered from the device outlet. Concentrations between $10^{3}-10^{5} \mathrm{CFU}$ of each microorganism, in pure or in mix cultures, were passed through the device containing the sponge to determine the Limit of Detection (LoD) of the methodology. In order to determine the uncaptured bacteria the collected sample was used for 10-fold serial dilutions and each one was plated and counted.

\subsubsection{Real sample testing}

To ensure the reliability of the results, the detection of L. monocytogenes and Salmonella spp. were tested spiking stainless steel surfaces in order to test the applicability of the developed methodology in food industry with real sample. To contaminate the surfaces, an ON culture prepared as describe in methods 2.2.3, was diluted and $10^{5} \mathrm{CFU}$ of a bacterial mixture was spread on the surface and let dry at room temperature. The bacteria were recovered with a cotton swab pre-moisturised in PBS with $0.01 \%$ of Tween 80 , and re-suspended in $2 \mathrm{~mL}$ of PBS by vortexing. One $\mathrm{mL}$ of the solution was passed through the device as specified in methods 2.2.3, and DNA extraction from the sponge, and downstream qPCR, were also performed as previously described in methods 2.2.4. The capture efficiency was also determine.

\section{Results and discussion}

\subsection{Sponge characterization for bacterial capturing}

To confirm the epoxide modification on the PDMS surface, FTIR spectrum were taken and presented in Fig. 3 a. The vibrational modes around 960, 1080, and $1195 \mathrm{~cm}^{-1}$ has been observed and are 
considered to arise from un-hydrolyzed ethoxy moieties in APTES $\left(-\mathrm{OCH}_{2} \mathrm{CH}_{3}\right)$. The peaks indicate

243 presence of APTES resulting in layer of APTES over the PDMS surface. The peaks at $1045 \mathrm{~cm}^{-1}$ and

$2441125 \mathrm{~cm}^{-1}$ are attributed to the presence of $\mathrm{Si}-\mathrm{O}-\mathrm{Si}$ and $\mathrm{Si}-\mathrm{O}$ moieties, respectively. These peaks also correspond to the thickness of the APTES adsorbed layer over the PDMS samples. A band of absorbance appears between 2800 and $3000 \mathrm{~cm}^{-1}$ which corresponds to the elongation of the $\mathrm{CH}$ bond which is proportional to the quantity of carbon included in the grafted molecules. The $\mathrm{CH}_{2}$ and $\mathrm{CH}_{3}$ groups appear towards 2900 and $2987 \mathrm{~cm}^{-1}$. The peak for $-\mathrm{NH}$ arise near 1500 confirms the presence of APTES. The peak around $3300 \mathrm{~cm}^{-1}$ has been observed showing presence of -OH bonds i.e., trapped water or moisture in the cured APTES film over the PDMS sample. Peaks around 2900-3000 presents additional peaks for binding of ApoH ligand.

To ensure proper surface modification of the sponge and the ligand binding, a Fluorescent dye Atto633 was used to allow the visualization of the ligand in the surface. The Atto-633 is a red fluorescent dye with an excitation and emission spectrum optimally at $633 \mathrm{~nm}$ and $657 \mathrm{~nm}$, respectively. A bright signal is obtain as the proof of attachment of dye molecules and presented that the surface modification is not only on the surface but also inside the sponge in cross section view (Fig. 3 b, c, d).

The SEM characterization of PDMS sponge is presented in Fig3, where Fig. 3e presents the mesoporous morphology of PDMS sponge generated due to the sacrificing salt particles in fabrication. This morphology shows various pores small and big connected each other. Due to this connectivity of porous structure PDMS sponge not make any resistance for the flow of sample solution. The Fig $3 \mathrm{f}$ presented the bacteria captured on top and inner surface of the sponge and a magnified view of the bacteria captured in PDMS sponge (Fig.3g). With these SEM images it's been observed that bacteria is been captured inside the holes of the sponges and other surfaces.

\subsection{Simplex and multiplexed bacterial capturing with ApoH protein}

\subsubsection{Capture efficiency in simplex}

The capacity of the sponge to retain the bacterial cells was evaluated testing two different bacteria, $L$. 
268

269

270

271

272

273

274

275

276

277

performed to confirm that the bacteria capturing is due to the interaction with ligand and not a nonspecific capturing in the pores of the sponge. Without any functionalization of the sponge it was observed a capture efficiency of $19.7 \pm 2.4$, when passed a bacteria concentration of $10^{4} \mathrm{CFU} / \mathrm{mL}$ into the device. This result show some capturing in the pores of the sponge, but the use of a ligand allow a great improvement in the capture of the bacteria. The results of the capture efficiency with sponges functionalized with ApoH protein are presented in Table 1 and Fig. 4 a. passing a pure culture of each bacteria separately into the device, the sponge obtained a capture efficiency above $88.6 \%$ and $78.6 \%$ for L. monocytogenes, and Salmonella spp., respectively. This results are comparable to others obtained with concentration methodologies for pathogen detection (Fakruddin, Hossain, and Ahmed 2017; Li et al. 2019).

\subsubsection{Capture efficiency in multiplex}

A mixture with the two pathogens was also tested and results showed a capture efficiency around 70 $\%$ for both bacteria when tested in the range of $10^{5} \mathrm{CFU}$ with higher deviation for $L$. monocytogenes (Table 1 and Fig. 4b). However an increase in this value with lower deviation was noticed when using a lower bacterial concentration (range of $10^{3} \mathrm{CFU}$ ). This fact may be due to the competition existing between the bacteria cells to bind to the ApoH protein when higher concentration is used, as when the capture efficiency for one pathogen increased, a decrease was visible for the other one (data not showed).

The deviation existing in the capture efficiency between replicates can also be explained by the fact that not all sponges have the same size, as the cutting was made manually and the porosity can also vary between sponges. This will influence the concentration of immobilized protein or antibody into the sponge and also the capacity of the flow to penetrate the sponge.

\subsubsection{Comparison between ApoH and Ab functionalization}

ApoH has the ability to bind to both, Gram-positive and Gram-negative, being a non-specific targeting, allowing the concentration of different pathogens at the same time. To test if the results provided by the use of this protein instead of a specific targeting is as efficient for bacterial capture, a 
294

295

296

297

298

299

300

301

302

303

304

305

306

307

308

309

310

311

312

313

314

315

316

317

318

319

specific anti-L. monocytogenes antibody was used to compare the ability to capture this pathogen.

Two L. monocytogenes concentration $10^{4}$ and $10^{3} \mathrm{CFU} / \mathrm{mL}$ were tested showing a capture efficiency of $78.3 \% \pm 12.3$ for $10^{4} \mathrm{CFU} / \mathrm{mL}$ and $76.8 \% \pm 4.1$ for $10^{3} \mathrm{CFU} / \mathrm{mL}$ using the specific antibody. Higher capture efficiency was observed using the ApoH protein, being $88.6 \% \pm 10.4$ and $88.8 \% \pm 0.5$, respectively. The comparison between these two different functionalization is represented in Fig. 4 c, showing an advantage in using the non-targeted approach.

\section{3.qPCR detection}

The detection by qPCR was performed to evaluate if the sponge methodology is compatible with this type of DNA-based detection, and additionally to allow the determination of the LoD of the full methodology. The amplification results are presented in Table 2 and Fig. 4 d. Bacteria concentration below the range of $10^{3} \mathrm{CFU}$ were not detectable by qPCR (data not shown). This results agree with the literature, being a limitation of the PCR (Simon, Gray, and Cook 1996). For Salmonella spp., the $10^{3} \mathrm{CFU} / \mathrm{mL}$ were achieved, however for L. monocytogenes, the lowest concentration which showed amplification in all replicates were $10^{4} \mathrm{CFU} / \mathrm{mL}$, separately and in mixture. L. monocytogenes is more difficult to lyse and for this reason a lower concentration of DNA is recovered from the sample and resulting in lower LoD. A possibility to overcome the limitation of the qPCR reaction and improve the sensitivity, is the addition of an enrichment step to the methodology in order to reach a detectable concentration of bacteria in the sample.

\subsection{Spiked samples}

Surface samples were inoculated to test the applicability of the methodology to capture and detect different pathogens at the same time in a real case scenario in the food industry. A mixture of both bacteria was spread on the surface, theed by the sponge as the capture efficiency showed to be higher than $96 \%$ for both cases (Table 1 and Fig. 4 b).

Previous steps performed before the introduction of the sample into the device can result in loss of bacteria. The sampling process from the surface and the elution of bacteria retained in the swab to the 
PBS solution is not $100 \%$ efficient, leading to an incomplete loading into the device, of the initial bacteria concentration spiked on the surface. This results in a lower bacterial concentration passed and retained in the sponge and increase the LoD when analysing surface samples. Besides this fact, the detection L. monocytogenes and Salmonella spp. was successfully accomplished by qPCR for both targets using a range of $10^{5} \mathrm{CFU}$ bacteria cells.

\section{Conclusion}

The results obtained in this study support the use of the methodology for multiplex pathogen detection, with a LoD between $10^{3}-10^{4}$, depending of the targeted pathogen, being comparable with other qPCR analysis results. This device showed high capture efficiency for both Gram positive and negative bacteria, above $70 \%$, and showed higher value using the ApoH protein than the specific anti- L. monocytogenes antibody (Figure 4c). The universal capturing efficiency of ApoH will reduce the cost of using bacteria specific antibody for capturing. The use of the device allowed a concentration of the bacteria and can be easily employed in the food industry for surface analysis. It is important to note that pre-treatment step of bacterial enrichment were excluded, and the preconcentration with the functionalized 3D PDMS sponge increased the analysis sensitivity. Additionally, the combination with qPCR offered great convenience for fast screening of multiplexed pathogen on-site. To conclude, this methodology represents a real advantage giving the possibility of full integration in a lab-on-ship system, as the DNA extraction and amplification step can be incorporated in a miniaturized devices, allowing the automatization of the analysis.

\section{Acknowledgement}

Authors acknowledge the FRONTHERA project (Frontiers of technology for theranostics of cancer, metabolic and neurodegenerative diseases) $\mathrm{n}^{\circ}$ NORTE-01-0145-FEDER-0000232. This work was supported by project Nanotechnology Based Functional Solutions (NORTE- 
(NORTE2020), under the PORTUGAL 2020 Partnership Agreement, through the European

Regional Development Fund.

\section{Reference}

C L C Bio-Qiagen. 2016. "CLC Sequence Viewer."

Cai, Shuowei, Bal Ram Singh, and Shashi Sharma. 2007. "Botulism Diagnostics: From Clinical Symptoms to in Vitro Assays." Critical Reviews in Microbiology 33(2):109-25.

Cai, Steven, Dirce Yorika Kabuki, Arnaldo Yoshiteru Kuaye, Theresa Gina Cargioli, Michael S. Chung, Rasmus Nielsen, and Martin Wiedmann. 2002. "Rational Design of DNA Sequence-

Coroneo, Valentina, Valentina Carraro, Nadhem Aissani, Adriana Sanna, Alessandra Ruggeri, Sara Based Strategies for Subtyping Listeria Monocytogenes." Journal of Clinical Microbiology 40(9):3319-25. Succa, Barbara Meloni, Antonella Pinna, and Clara Sanna. 2016. "Detection of Virulence Genes and Growth Potential in Listeria Monocytogenes Strains Isolated from Ricotta Salata Cheese." Journal of Food Science 81(1):M114-20.

Dao, Thuy Nguyen Thi, Jaeyun Yoon, Choong Eun Jin, Bonhan Koo, Kyudong Han, Yong Shin, and Tae Yoon Lee. 2018. "Rapid and Sensitive Detection of Salmonella Based on Microfluidic Enrichment with a Label-Free Nanobiosensing Platform.” Sensors and Actuators B: Chemical 262:588-94.

Dewey-Mattia, Daniel, Karunya Manikonda, Aron J. Hall, Matthew E. Wise, and Samuel J. Crowe. 2018. "Surveillance for Foodborne Disease Outbreaks — United States, 2009-2015." MMWR. Surveillance Summaries 67(10):1-11.

EFSA and ECDC. 2018. "The European Union Summary Report on Trends and Sources of Zoonoses, Zoonotic Agents and Food $\square$ borne Outbreaks in 2017.” EFSA Journal 16(12).

Fakruddin, Md, Md Nur Hossain, and Monzur Morshed Ahmed. 2017. "Evaluation of Microplate Immunocapture Method for Detection of Vibrio Cholerae, Salmonella Typhi and Shigella Flexneri from Food.” BMC Microbiology 17(1):189.

Garrido-Maestu, Alejandro, Sarah Azinheiro, Joana Carvalho, and Marta Prado. 2018. "Rapid and Sensitive Detection of Viable Listeria Monocytogenes in Food Products by a Filtration-Based Protocol and QPCR.” Food Microbiology 73:254-63. 
Garrido-Maestu, Alejandro, Pablo Fuciños, Sarah Azinheiro, Joana Carvalho, and Marta Prado. 2017.

"Systematic Loop-Mediated Isothermal Amplification Assays for Rapid Detection and Characterization of Salmonella spp., Enteritidis and Typhimurium in Food Samples.” Food Control 80:297-306.

Garrido $\square$ Maestu, Alejandro, Sarah Azinheiro, Joana Carvalho, and Marta Prado. 2019. "Combination of Immunomagnetic Separation and Real-Time Recombinase Polymerase Amplification (IMSQRPA) for Specific Detection of Listeria Monocytogenes in Smoked Salmon Samples.” Journal of Food Science.

Gorgannezhad, Lena, Helen Stratton, and Nam-Trung Nguyen. 2019. "Micromachines MicrofluidicBased Nucleic Acid Amplification Systems in Microbiology."

Kawasaki, Susumu, Naoko Horikoshi, Yukio Okada, Kazuko Takeshita, Takashi Sameshima, and Shinichi Kawamoto. 2005. "Multiplex PCR for Simultaneous Detection of Salmonella spp., Listeria monocytogenes, and Escherichia coli O157:H7 in Meat Samples.” Journal of Food Protection 68(3):551-56.

Li, Zhiming, Jinyuan Ma, Jun Ruan, and Xuan Zhuang. 2019. "Using Positively Charged Magnetic Nanoparticles to Capture Bacteria at Ultralow Concentration." Nanoscale Research Letters 14(1):195.

Liu, Jiayu, Ibrahem Jasim, Zhenyu Shen, Lu Zhao, Majed Dweik, Shuping Zhang, and Mahmoud Almasri. 2019. “A Microfluidic Based Biosensor for Rapid Detection of Salmonella in Food Products" edited by C. Li. PLOS ONE 14(5):e0216873.

Oravcová, K., T. Kuchta, and E. Kaclíková. 2007. “A Novel Real-Time PCR-Based Method for the Detection of Listeria monocytogenes in Food." Letters in Applied Microbiology 45(5):568-73.

Simon, Moyra C., David I. Gray, and Nigel Cook. 1996. DNA Extraction and PCR Methods for the Detection of Listeria monocytogenes in Cold-Smoked Salmon. Vol. 62.

Sousa, Cláudia, Diana Sequeira, Yury V. Kolen'Ko, Inês Mendes Pinto, and Dmitri Y. Petrovykh. 2015. "Analytical Protocols for Separation and Electron Microscopy of Nanoparticles Interacting with Bacterial Cells." Analytical Chemistry 87(9):4641-48.

Untergasser, Andreas, Ioana Cutcutache, Triinu Koressaar, Jian Ye, Brant C. Faircloth, Maido Remm, and Steven G. Rozen. 2012. "Primer3-New Capabilities and Interfaces." Nucleic Acids Research 40(15).

Vidic, Jasmina, Priya Vizzini, Marisa Manzano, Devon Kavanaugh, Nalini Ramarao, Milica Zivkovic, Vasa Radonic, Nikola Knezevic, Ioanna Giouroudi, and Ivana Gadjanski. 2019. 
"Point-of-Need DNA Testing for Detection of Foodborne Pathogenic Bacteria." Sensors 19(5):1100.

Vinayaka, Aaydha C., Tien A. Ngo, Krishna Kant, Pia Engelsmann, Vivek P. Dave, Mohammad-Ali Shahbazi, Anders Wolff, and Dang D. Bang. 2019. "Rapid Detection of Salmonella Enterica in Food Samples by a Novel Approach with Combination of Sample Concentration and Direct PCR." Biosensors and Bioelectronics 129:224-30.

Wang, Yun and Timothy V Duncan. 2017. "Nanoscale Sensors for Assuring the Safety of Food Products." Current Opinion in Biotechnology 44:74-86.

$417 \quad$ Fig 1.

(A)

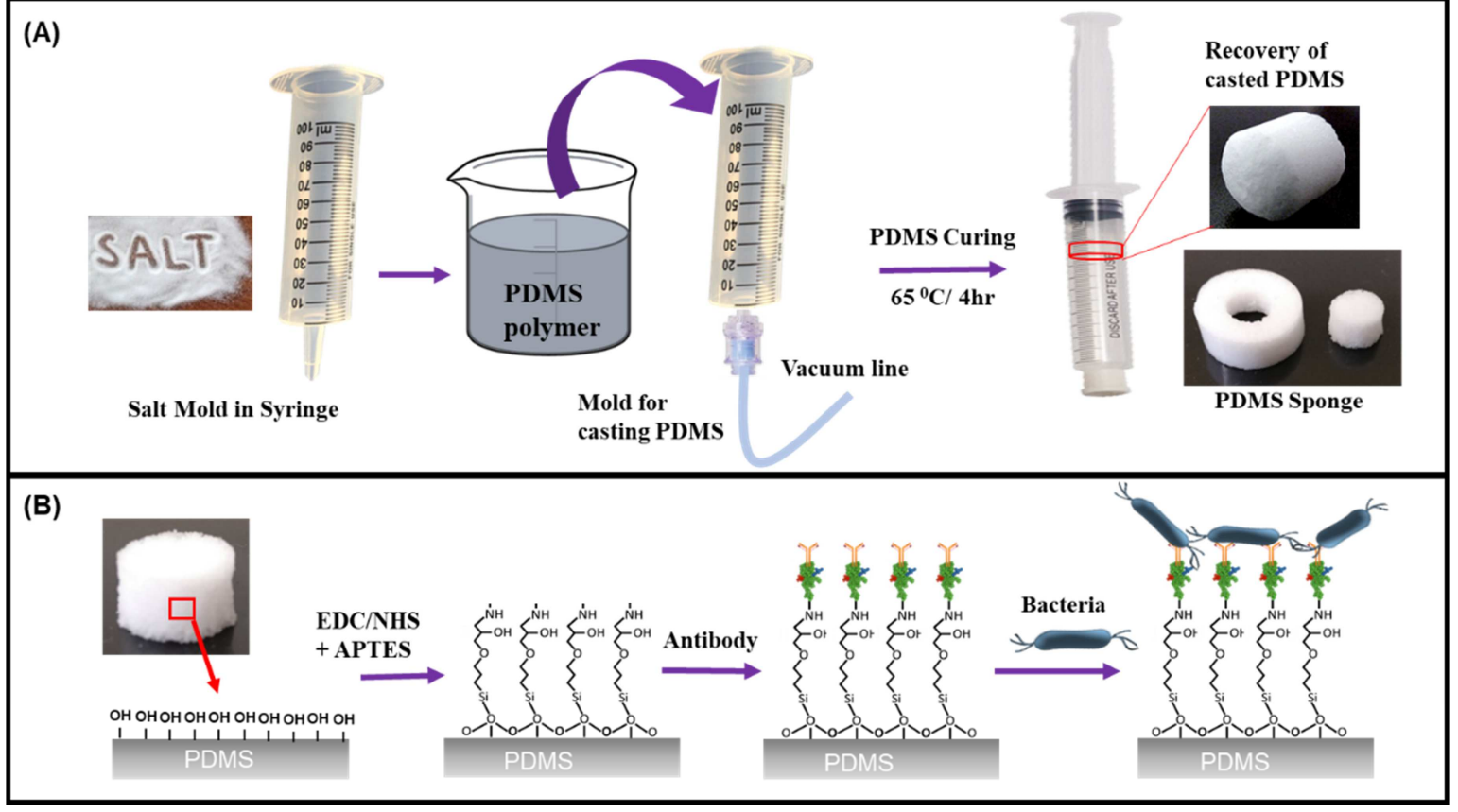

418

419 Fig. 1: (A) Schematic presentation of fabrication process of PDMS sponge casting and recovery using salt template. (B) Surface modification over the PDMS sponge for capturing of bacteria. 


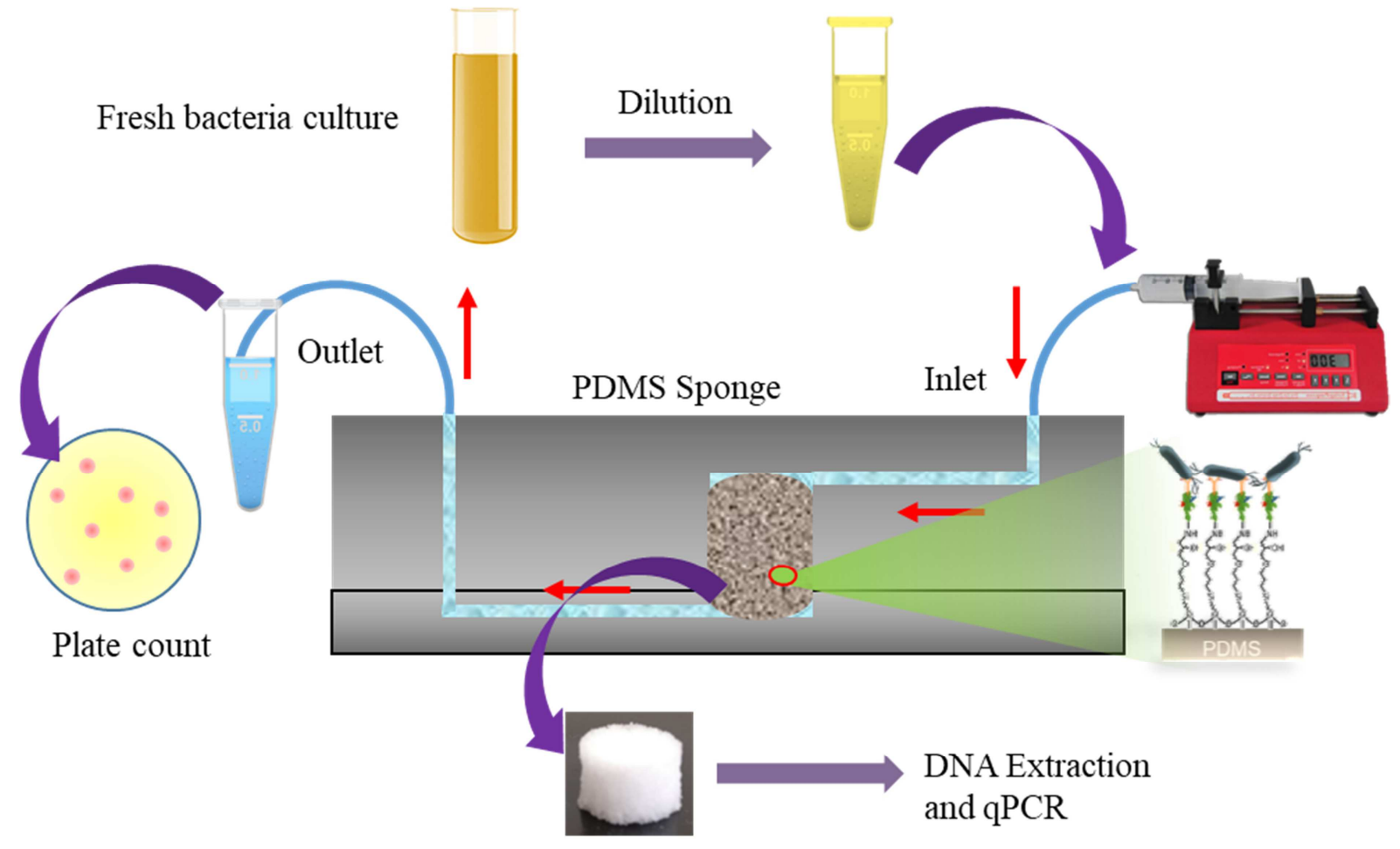

424 Fig. 2: Schematic presentation of the microfluidic chip for capturing the bacteria.

$425 \quad$ Fig 3. 

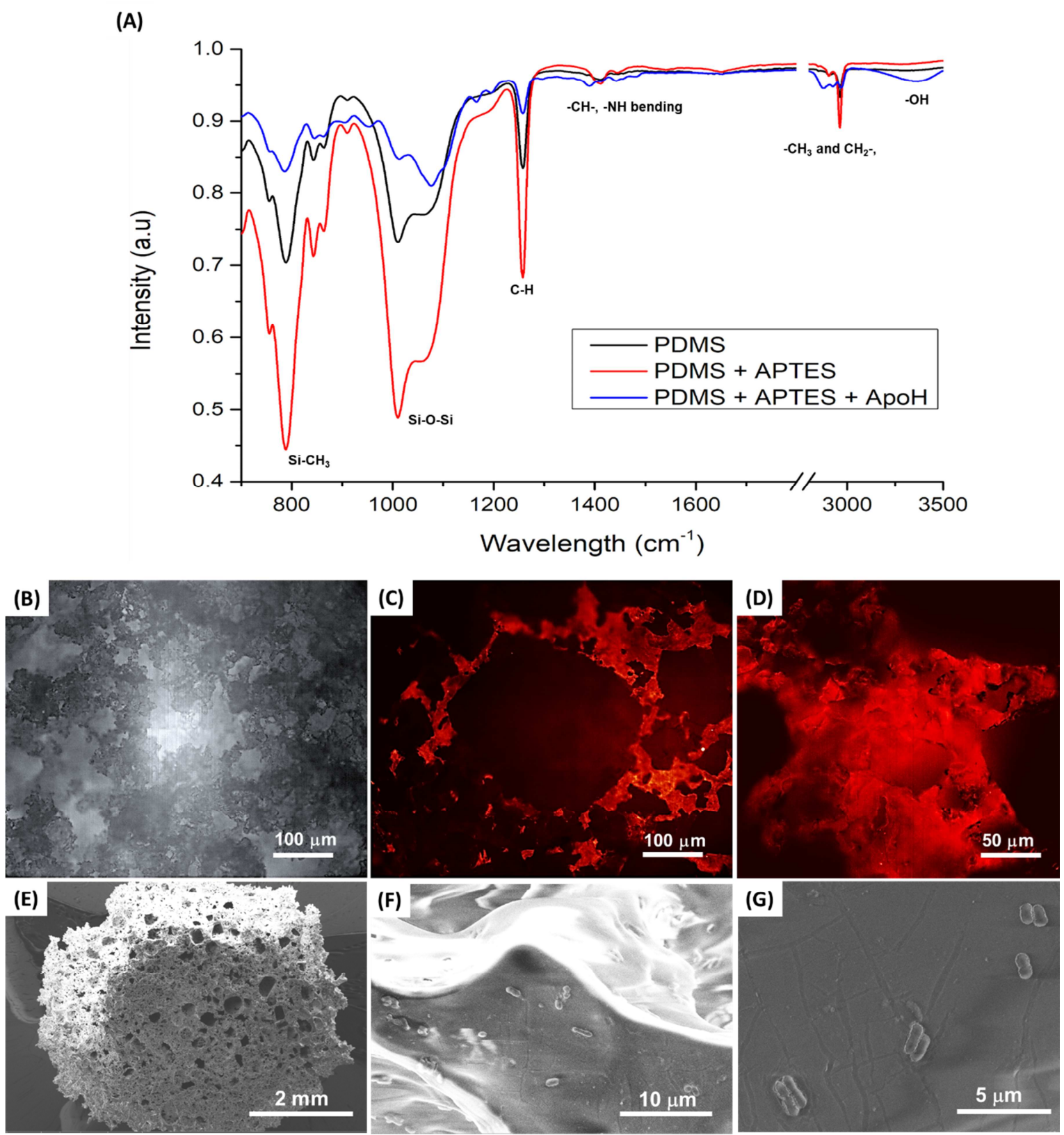

(G)

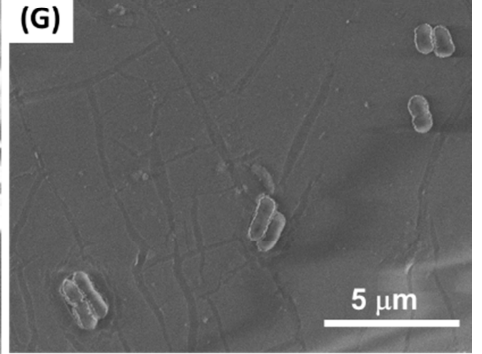

Fig. 3: (A) Fourier Transform Infrared Spectroscopy (FTIR) spectra for different stages of surface modification (B) An optical image of the sponge before fluorescence imaging (C) Fluorescent image of PDMS sponge after surface modification (D) a cross-section view of the PDMS sponge to confirm the surface modification inside sponge pores. SEM characterization of PDMS sponge is presented after bacterial capturing (E) bare PDMS Sponge with its mesoporous morphology (F) sponge surface after L. monocytogenes capturing $(\mathrm{G})$ magnified view of bacteria on sponge surface. 
438
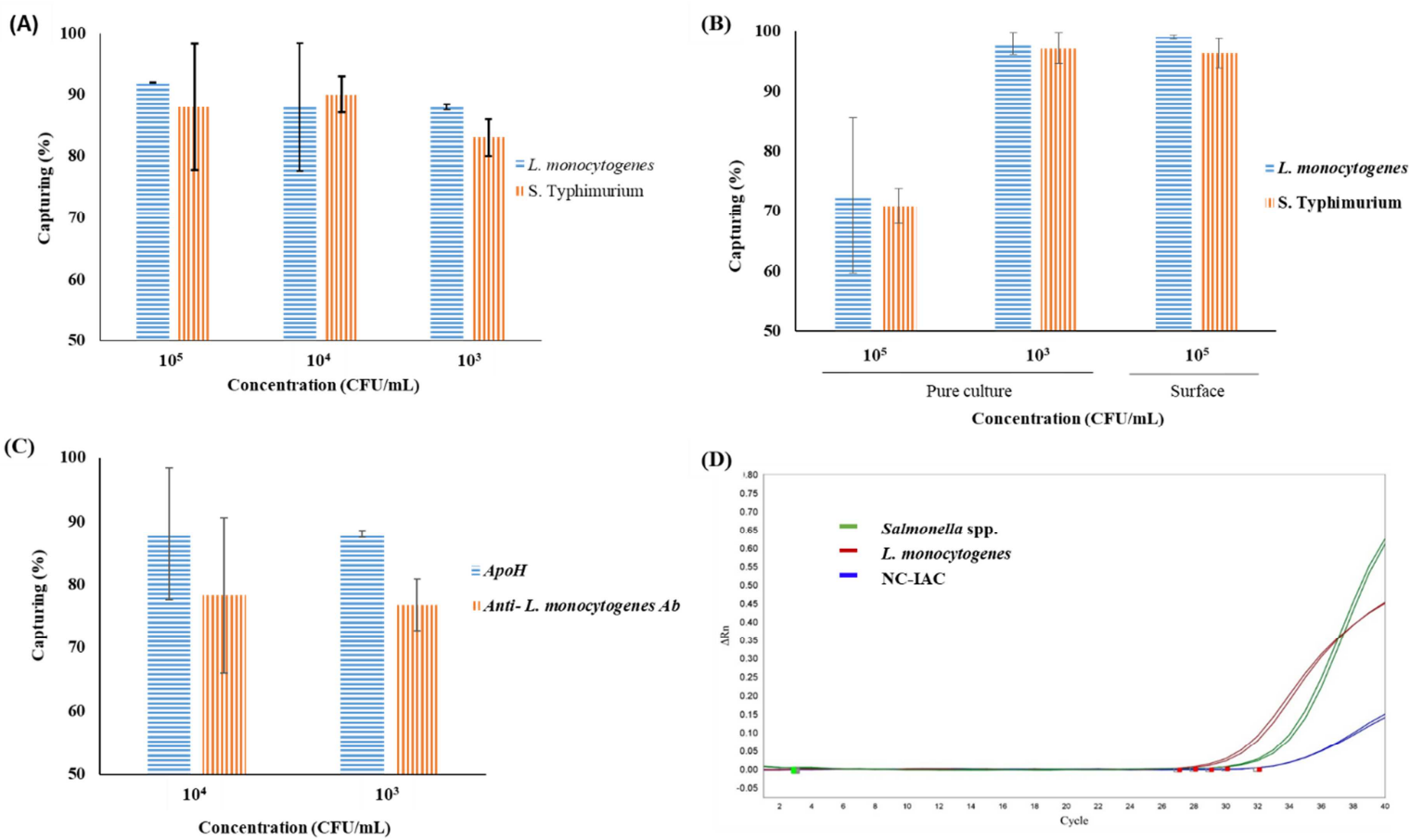

Fig. 4: Graphical representation of capture efficiency for L. monocytogenes and Salmonella spp. using the PDMS sponge into the device in simplex (A) and multiplex using pure culture or passing a surface sample (B). Comparison of capture efficiency using PDMS sponge functionalized with ApoH protein and Anti- L. monocytogenes specific antibody (C). And qPCR amplification plots (D) detecting L. monocytogenes and Salmonella spp. in a concentration of $10^{5} \mathrm{CFU} / \mathrm{mL}$. NC-IAC was included in the reaction to detect inhibition and avoid false negative results due to reaction inhibition. 
Table 1

Table 1 - Capturing efficiency in pure and mixed culture in the sponge, functionalized with ApoH protein

\begin{tabular}{l|ccc|cc|c}
\hline \multicolumn{1}{c|}{ Bacteria } & \multicolumn{3}{c|}{ Pure culture } & \multicolumn{2}{c|}{ Mixed culture } & Surface \\
concentration & $10^{5}$ & $10^{4}$ & $10^{3}$ & $10^{5}$ & $10^{3}$ & $10^{5}$ \\
\hline L.monocytogenes & $92.3 \pm 0.1$ & $88.6 \pm 10.4$ & $88.8 \pm 0.5$ & $72.6 \pm 12.9$ & $98.7 \pm 1.8$ & $99.7 \pm 0.3$ \\
S. Typhimurium & $78.6 \pm 10.3$ & $90.5 \pm 3.0$ & $83.8 \pm 3.0$ & $70.9 \pm 2.9$ & $97.2 \pm 2.5$ & $96.4 \pm 2.5$ \\
\hline
\end{tabular}

Capture efficiency is expressed as \% and the bacterial concentration in CFU/ $\mathrm{mL}$

\section{Table 2}

Table $2-$ qPCR results

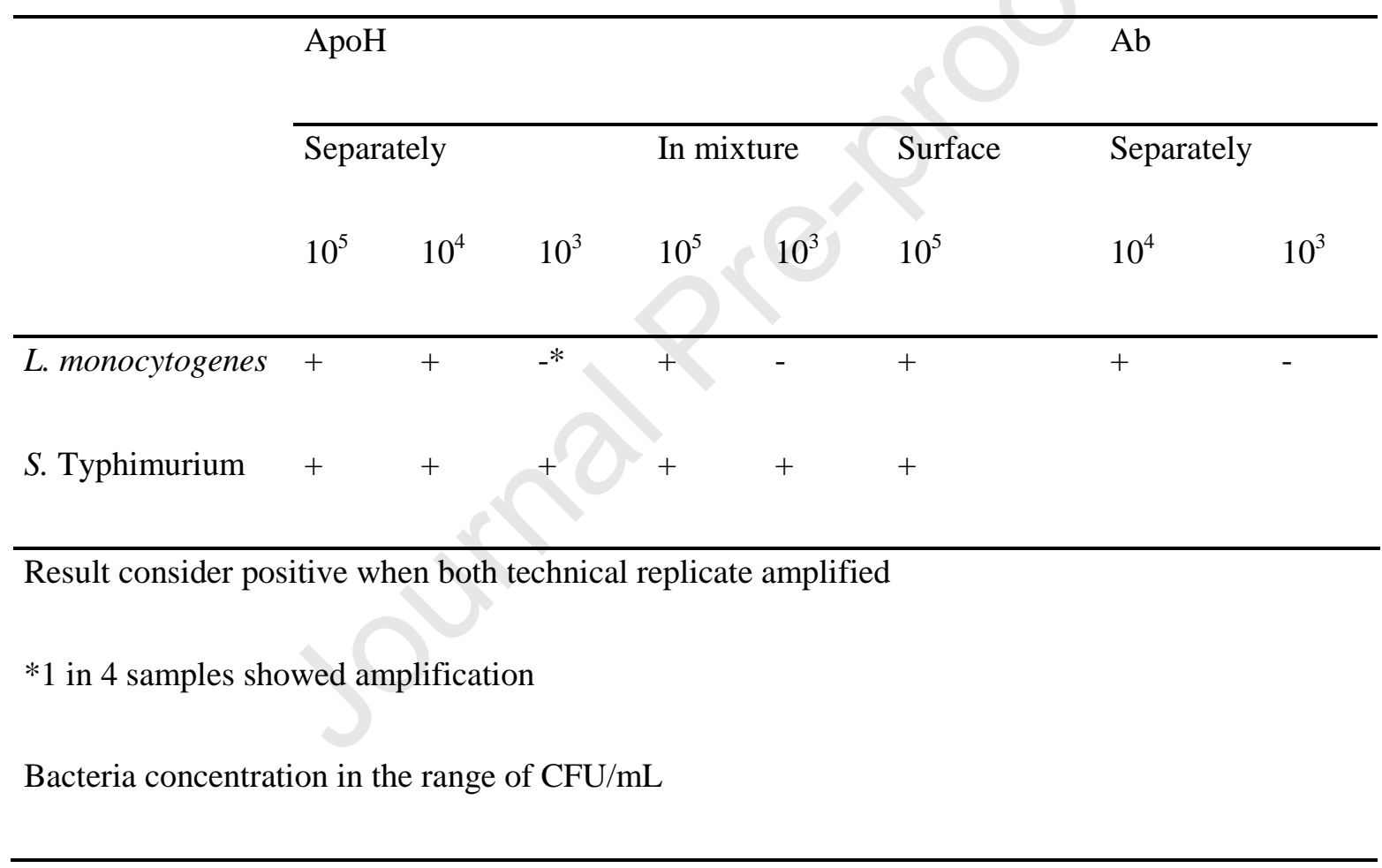


Supplementary material

Table S1. Strain list and multiplex qPCR results for actA genes

\begin{tabular}{llll}
\hline Bacterium & Source & $\mathrm{N}$ & actA \\
\hline L. monocytogenes & WDCM00021 & 1 & + \\
L. monocytogenes & Food & 17 & + \\
L. ivanovii & WDCM00018 & 1 & - \\
L. innocua & WDCM00017, CECT 5376, 4030; & 6 & - \\
E. faecalis & CUP 1141, 1325, 2110 & & - \\
C. coli & WDCM00009 & 1 & - \\
E. coli & UM & 1 & - \\
Salmonella spp. & WDCM00013 & 1 & - \\
\hline
\end{tabular}

Evaluation of the inclusivity and exclusivity of the qPCR reaction using actA primers in simplex. All L. monocytogenes strains were correctly identified and all non-target bacteria were not detected in the reaction.

$\mathrm{N}$ : number of strains;

471

472

473

474

475

476

477

478

479 
Table S2- Primers and probes used

Sequence 5'-3' Concentration used

\begin{tabular}{|c|c|c|}
\hline $\operatorname{act} A \mathrm{~F}$ & TTAAGACTTGCTTTGCCAGAGAC & \\
\hline $\operatorname{act} A \mathrm{R}$ & GGTGGTGGAAATTCGAATGAGC & \\
\hline actA $\mathrm{P}$ & $\begin{array}{l}{ }^{\text {CY5-AATGCTCCT }}{ }^{- \text {TAO- }} \text { GCTACATCGGAACCGA } \\
\text { IAbRQSp }\end{array}$ & $150 \mathrm{nM}$ \\
\hline
\end{tabular}

\begin{tabular}{lll}
\hline $\operatorname{ttr} \mathrm{F}$ & GGCTAATTTAACCCGTCGTCAG & \multirow{2}{*}{$200 \mathrm{nM}$} \\
\cline { 1 - 2 } $\operatorname{ttr} \mathrm{R}$ & GTTTCGCCACATCACGGTAGC & \\
\hline $\operatorname{ttr} \mathrm{P}$ & NED-AAGTCGGTCTCGCCGTCGGTG $^{-\mathrm{MGBNFQ}}$ & $150 \mathrm{nM}$
\end{tabular}

NC-IAC F $\quad$ TTAAGACTTGCTTTGCCAGAGAC

NC-IAC R GGTGGTGGAAATTCGAATGAGC $100 \mathrm{nM}$

$\begin{array}{ll}\text { NC-IAC P } & \text { YY-AGT GGC GGT }^{- \text {ZEN- }} \text { GAC ACT GTT GAC CT } \\ & -\end{array}$ IABkFQ

YY (Yakima Yellow), IAbRQSp and IABkFQ (Iowa Black ${ }^{\circledR} F Q$ and RQ-Sp), ZEN and TAO (secondary, internal quencher) are trademarks from IDT. 


\section{$\operatorname{act} \mathbf{A}$}

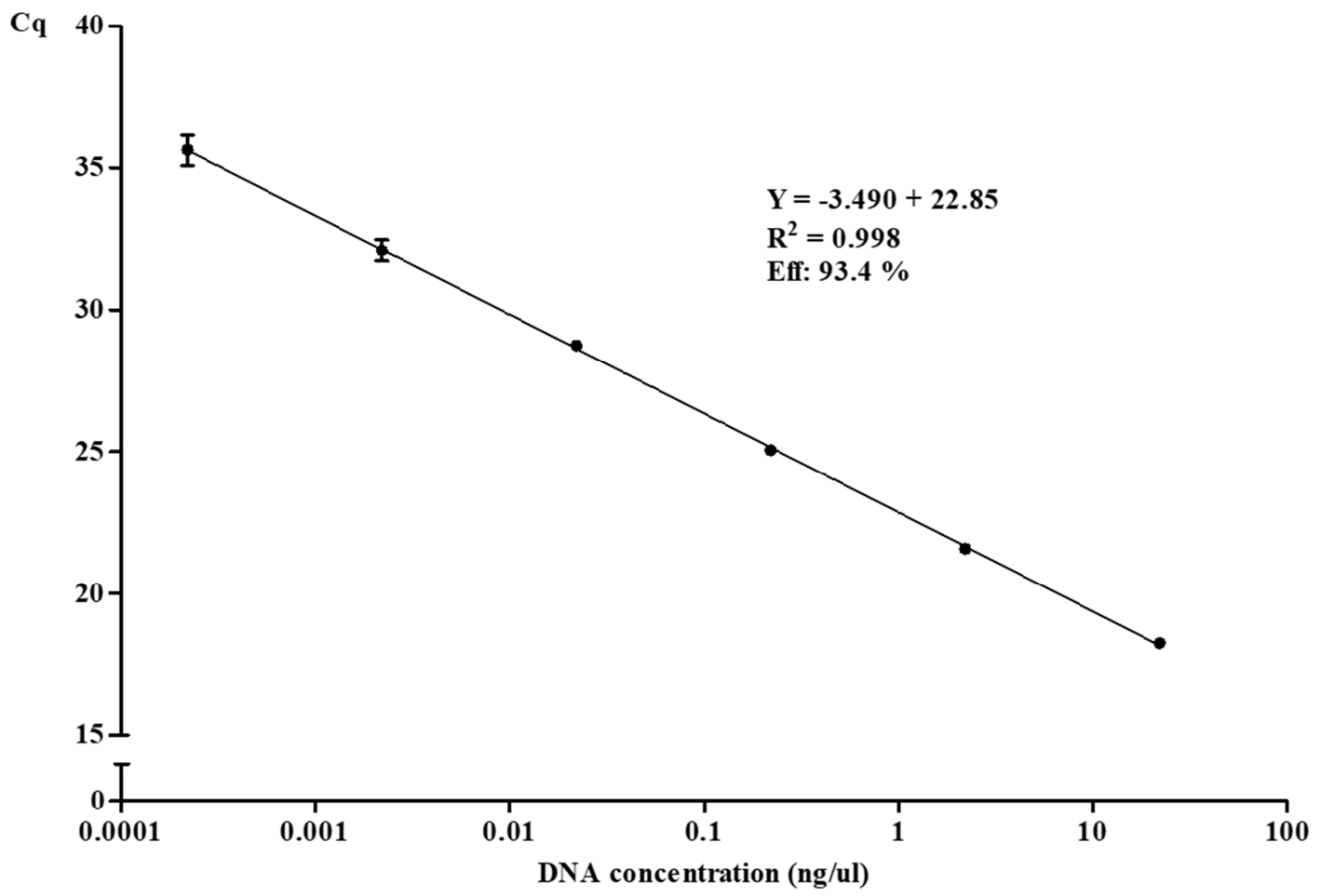

481

482 Fig. S1: qPCR amplification efficiency of L. monocytogenes, targeting actA and NC-IAC 483 simultaneously. Curve was obtained by three replicates of ten-fold serial dilutions of a pure DNA 484 extract. 


\section{Highlights}

- A mesoporous PDMS sponge is used for multiplexed foodborne pathogen capturing.

- Universal binding ligand (Apo-H) is used for multiplexed bacterial capturing.

- Comparisons of bacteria capturing efficiency against slandered Antibody using qPCR. 


\section{Declaration of interests}

$\bigotimes$ The authors declare that they have no known competing financial interests or personal relationships that could have appeared to influence the work reported in this paper.

$\square$ The authors declare the following financial interests/personal relationships which may be considered as potential competing interests:

Krishna Kant

30/09/2019 